\title{
Ultrasound Mediated Microbubbles Destruction Assists Dual Delivery of Beta-amyloid Antibody and NSCs to Restore Neural Function in Transgenic Mice of Alzheimer's Disease
}

qiong Zhu

Army Medical University

hai Cui

Army Medical University

yiyi Liao

Army Medical University

xue Guan

Army Medical University

ying $\mathrm{He}$

Army Medical University

yani Rong

Army Medical University

yi Zhang

Army Medical University

chun Li

Army Medical University

zheng Liu

Army Medical University

yali xu ( $\nabla$ xuyali1976@163.com )

Army Medical University https://orcid.org/0000-0002-6311-8757

Research

Keywords: Alzheimer's disease, Ultrasound mediated microbubble destruction, Beta-amyloid, Neural stem cells, Blood-brain barrier

Posted Date: May 11th, 2020

DOI: https://doi.org/10.21203/rs.3.rs-27241/v1 
License: (c) (i) This work is licensed under a Creative Commons Attribution 4.0 International License. Read Full License 


\section{Ultrasound Mediated Microbubbles Destruction Assists Dual}

Delivery of Beta-amyloid Antibody and NSCs to Restore Neural

\section{Function in Transgenic Mice of Alzheimer's Disease}

AUTHORS: Qiong Zhu ${ }^{1}$, Hai Cui ${ }^{1,2}$, Yiyi Liao ${ }^{1}$, Xue Guan ${ }^{1}$, Ying He ${ }^{1}$, Yani Rong ${ }^{1}$,

Yi Zhang ${ }^{1}$, Chun $\mathrm{Li}^{1}$, Zheng Liu ${ }^{1}$ and Yali $\mathrm{Xu}^{1}$

\section{Affiliations}

1.Department of Ultrasound, Xinqiao Hospital, Army Medical University

(Third Military Medical University), Chongqing, China.

2.Department of Ultrasound, Armed Police Force Hospital, Sichuan, China.

\section{Corresponding author}

Xu Yali, M.D., Ph.D.

Department of Ultrasound, Xinqiao Hospital,

Army Medical University, Chongqing 400037, China.

Email: xuyali1976@163.com

Telephone: $86-23-68755681$ 


\section{Abstract}

Background: To explore the feasibility, efficacy and safety of ultrasound mediated microbubbles destruction ( UMMD ) assisted dual delivery of $\beta$-amyloid antibody loaded by microbubbles $\left(\mathrm{MB}_{\mathrm{A} \beta}\right)$ and neural stem cells (NSCs) on Alzheimer's disease ( $\mathrm{AD}$ ).

Methods: 27 APP/PS1 double transgenic mice and 33 wild-type mice were used. The dual delivery of $\beta$-amyloid antibody and NSCs group (US+MB $\mathrm{M}_{\mathrm{A}}+\mathrm{NSCs}$ ), single delivery of $\beta$ amyloid antibody group (US+MB $\mathrm{MB}_{\mathrm{A}}$ ), US+MB group, Control group and Wild group, were involved in the experiment. $\mathrm{MB}_{\mathrm{A} \beta}$ or $\mathrm{MB}$ were injected via the tail vein, followed by NSCs or saline administration and exposed to ultrasound once a week for four times. The survival of NSCs was detected with the in vivo imaging method. Mice in each group were used for behavioral function evaluation and the pathology tests. Brain samples were used to detect $\beta$ amyloid deposition, BDNF and synaptophysin expression.

Results: BBB was opened by UMMD with an opening time about $10 \mathrm{~h}$. The transplanted NSCs survived in AD brain for no more than $72 \mathrm{~h}$. The learning and spatial memory function was significantly improved in the $\mathrm{US}+\mathrm{MB}_{\mathrm{A} \beta}+\mathrm{NSCs}$ group, $\mathrm{US}+\mathrm{MB}_{\mathrm{A} \beta}$ group came second. Immunochemistry results showed amyloid plaques reduction in the US+MB $\mathrm{M}_{\mathrm{A}}+\mathrm{NSCs}$ group at the cortex and hippocampus. Higher level of BDNF was demonstrated in the US+MB $\mathrm{A}_{\mathrm{A}}+\mathrm{NSCs}$ group than the US+MB$B_{A \beta}$ group and the Control group with Western Blot and immunofluorescence examination, but synaptophysin remained no significant changes.

Conclusions: UMMD assisted combined delivery of $\beta$-amyloid antibody and NSCs to AD mice brain can help to clear the $\mathrm{A} \beta$ peptide, increase BDNF level and restore the impaired neural function, which was superior to $\beta$-amyloid antibody delivery group. Therefore, the combined 
45

46

47

48

49

50

51

52

53

54

55

56

57

58

59

60

61

62

63

64

66

65

targeted delivery assisted by UMMD strategy may be a promising and safe method on treating AD.

Keywords: Alzheimer's disease; Ultrasound mediated microbubble destruction; Beta-amyloid;

Neural stem cells; Blood-brain barrier

(1)

50

51

52

3

54

55

56

7

58

59

60

61

62

63

64




\section{Background}

Alzheimer's disease ( AD ) is the leading cause of dementia in the aged population and is defined as a progressive neurodegenerative disorder. The pathological hallmarks are the $\beta$ amyloid $(\mathrm{A} \beta)$ plaques and neurofibrillary tangles, which have become the most appropriate targets for the therapy of $\mathrm{AD}[1,2]$. However, most of the therapeutants can hardly enter the brain owing to the blockage of blood brain barrier (BBB). Ultrasound (US) mediated microbubbles (MB) destruction (UMMD) induced BBB opening has provided a potential delivery approach for the therapeutic agents to enter the brain [3]. Several studies have focused on the reduction or clearance of $A \beta$ plaque using US [4-7]. Nisbet and $\mathrm{Xu}$ have reported that both $A \beta$ and Tau aggregates can be effectively cleared and the neuronal function has been restored with US [8-10]. Previous studies have proved that mesenchymal stem cells have been targeted and successfully homed into brain under UMMD induced BBB opening, which resulted in the improvement on neuronal loss and the cognitive and behavioral function in brain ischemic rats $[11,12]$. Other studies reported similar results following neuron stem cells (NSCs) implantation in AD animals $[13,14]$. Since $A \beta$ peptide is released into the extracellular space whereas tau is distributed in the neuron and axon, $A \beta$ may be more appropriate than Tau as a therapeutic target to be delivered into brain under UMMD in our research. Aiming to strengthen the recovery on the neuronal function and the clearance of $\mathrm{A} \beta$ deposits, a dual delivery of both the NSCs and A $\beta$ antibody under UMMD was performed in APP/PS1 $(+)$ transgenic AD mice in this study.

\section{Materials and Methods}

\section{Experimental Animals}


APP/PS1 double transgenic mice, B6/JNju-Tg ( APPswe ,PSEN1dE9 )/Nju, were purchased from Animal Research Center of Nanjing University. Mice were housed in a specific pathogen free environment with a $12 \mathrm{~h}$ light-dark cycle at a constant temperature about $24{ }^{\circ} \mathrm{C}$. Twentyseven 9 12-month-old APP/PS1 (+) mice and thirty-three age-matched wild-type mice, weighed 25-30 g, were used in this study.

All procedures involving animals were approved by the Institutional Animal Care and Use Committee of Army Medical University and were performed in accordance with the guidelines from the Chinese Animal Welfare Agency. The number of animals used was minimized. All mice were anesthetized intraperitoneally with 2, 2, 2-tribromoethanol (1.2\%, $10 \mathrm{ml} / \mathrm{Kg}$, SigmaAldrich, St. Louis, MO, USA), placed in a prone position with arms and legs immobilized. The hair of the mice skull was removed using depilatory creams to expose the parietal.

\section{Diagnostic US parameters}

A linear array probe (X4-12L) of a VINNO 70 diagnostic US scanner (Vinno Technology Co.Ltd, SuZhou, China) was used as MB trigger to open the BBB of mice in this study. For image-guided therapy, the system was modified, and the mode was called V-flash with adjustable therapeutic parameters and treatment area. In this study, mice were sonicated for 5 min with $3 \mathrm{MHz}$ frequency, $50 \mathrm{~Hz}$ pulse repetition frequency and 26 cycles pulse length line by line at a density of $32 / \mathrm{cm}$, and the transducer was positioned anterior to the connection line of ears to ensure the beams focused at the targeted area (Fig. 1).The MI value was around 0.8, the transmitting/intermittent time was $0.62 / 2 \mathrm{~s}$, and the focal depth was set at $1 \mathrm{~cm}$.

\section{The preparation and characterizations of $\beta$-amyloid antibody-loaded and unloaded MB}

The unloaded MB were prepared according to the protocols of the previous study [15]. And 
111 the concentration of MB is (2-9) $\times 10^{9} / \mathrm{ml}[16]$. The $\beta$-amyloid antibody loaded microbubbles $112\left(\mathrm{MB}_{\mathrm{A} \beta}\right)$ were prepared by an electrostatic absorption method with the purified anti- $\beta$-amyloid, 1-42 (mouse monoclonal, BioLegend, Inc. San Diego, CA) and MB. $10 \mu 1$ of 1-42 anti- $\beta-$ amyloid was added into the lyophilized lipid suspension of MB and incubated together for $1 \mathrm{~h}$ at room temperature, followed by the infusion of $\mathrm{C}_{3} \mathrm{~F}_{8}$ in each vial. After agitation, $\mathrm{MB}$ labeled with $\beta$-amyloid antibody was formed. In this study, both $\mathrm{MB}$ and $\mathrm{MB}_{\mathrm{A} \beta}$ were diluted for 100 times with normal saline (NS). $\mathrm{MB}_{\mathrm{A} \beta}$, the lipid membranes of $\mathrm{MB}_{\mathrm{A} \beta}$ were labeled with1,1-dioctadecyl-3,3,3,3tetramethylindocarbocyanine perchlorate ( $1 \mu \mathrm{l}$, DiI, Yeasen Co. Ltd., Shanghai, China), and the $\beta$-amyloid antibody was labeled with FITC-labeled Goat Anti-Mouse Ig-G $(1 \mu$, Beyotime Institute of Biotechnology, China). Both DiI and FITC were incubated for $45 \mathrm{~min}$ at room temperature. After incubation, the mixtures were washed for 3 times by centrifugation flotation at $300 \mathrm{rpm}$ for $3 \mathrm{~min}$ to remove the extra DiI and FITC. The fluorescent microscopy of the labeled $\mathrm{MB}_{\mathrm{A} \beta}$ was observed by confocal laser scanning microscope (Leica TCS SP5, Wetzlar, Germany).

\section{BBB Opening procedure and histological examination} anterior to the line of ears and at the rear of eyes. To confirm the BBB opening efficacy and 
sonication, $300 \mu \mathrm{MB}$ or $\mathrm{MB}_{\mathrm{A} \beta}$ was administrated intravenously through the tail vein continuously to ensure there were enough cavitation nuclei in the mice brain. Both twodimensional and contrast-enhanced US imaging modality were performed to confirm the targeted irradiation area. After US exposure, EB was injected at a dose of $50 \mathrm{mg} / \mathrm{Kg}$ via the tail hour after EB injection, the mice were transcardially perfused (30 ml NS) and sacrificed. Then, the gross views of the harvested brains were observed to access the BBB opening capability and opening time duration. perfused (30 $\mathrm{ml} \mathrm{NS}$ followed by $10 \mathrm{ml} 4 \%$ paraformaldehyde) and sacrificed immediately after the sonication. Then the brains were harvested and immersed in 4\% paraformaldehyde for 24 staining was performed to assess the potential brain damages. Images were visualized with a light microscope (BX63, Olympus, Japan).

\section{The preparation and characterization of Neural stem cells}

flasks containing DMEF/F12 (Gibco, NY, USA) serum-free medium supplemented with 2\%

B27 supplement (50×,Gibco, NY, USA), 1\% recombinant murine epidermal growth factor 

were dissociated to get single cell using StemPro Accutase cell dissociation reagent (Gibco, NY, USA) for passaging or transplantation. NSCs at the third to fifth passage were used in this study.

\section{In vivo imaging of the luciferase labeled NSCs}

To evaluate whether the NSCs could pass the pulmonary circulation and home to the brain or not, a whole-animal imaging was performed to detect bioluminescent signals of NSCs using the in vivo imaging Spectrum system (IVIS, Xenogen, Alameda, CA, USA). Images were acquired and analyzed by Living Image 4.5 (Xenogen, Alameda, CA, USA).

For the in vivo tracking, NSCs at the $3^{\text {rd }}$ passage was labeled with firefly luciferase (fLuc) via lentivirus (GeneChem, Co. Ltd. Shanghai, China) transfection according to the manufacturer's protocol and seeded in 96-well plates at $5 \times 10^{4}$ cells $/ \mathrm{ml}$. Seventy-two hours after transfection, NSCs encoding fLuc were sorted by the puromycin at different concentration passaging.

The Luciferase labeled NSCs (fLuc-NSCs, $200 \mu 1,2 \times 10^{6}$ cells $/ \mathrm{ml}$ ) were injected into the APP/PS1 $(+)$ mice $(n=3)$ via the tail vein after BBB opening. The in vivo images of NSCs were captured $15 \mathrm{~min}$ after intraperitoneal injection of D-luciferin substrate $(10 \mu \mathrm{L} / \mathrm{g}$, Solarbio

172 Science \& Technology Co., Ltd., Beijing, China) to observe the distribution and survival of

173 fLuc-NSCs in mice brain every $24 \mathrm{~h}$ until no bioluminescent signals were detected.

\section{Treatment protocol}

175 The treatment protocol was described in Table 1. Mice were randomly divided into five groups and the treatment protocol of each group was as follows: US $+\mathrm{MB}_{\mathrm{A} \beta}+\mathrm{NSC}$ group 
(UMMD assisted dual delivery group): during US exposure, the V-flash mode was switched on and the treatment center was focused at the hippocampus, $300 \mu$ diluted $\mathrm{MB}_{\mathrm{AB}}\left(2-9 \times 10^{7} / \mathrm{ml}\right)$ was administrated into the APP/PS1 (+) mice within 5 min, after the US exposure, $200 \mu 1$ NSCs $\left(2 \times 10^{6}\right.$ cells $\left./ \mathrm{ml}\right)$ were infused. $U S+\mathrm{MB}_{\mathrm{A} \beta}$ group (single delivery of $\mathrm{A} \beta$ antibody): $300 \mu 1$ diluted $\mathrm{MB}_{\mathrm{A} \beta}$ and $200 \mu \mathrm{NS}$ was given to the APP/PS1 (+) mice under US exposure for $5 \mathrm{~min}$. US+MB group: $300 \mu \mathrm{MB}$ and $200 \mu \mathrm{l}$ NS infusion with US exposure on APP/PS1 (+) mice. Control group: $500 \mu 1 \mathrm{NS}$ was administrated into the APP/PS1 (+) mice with a sham US exposure. Wild group: $500 \mu \mathrm{NS}$ was administrated into the APP/PS1 (-) mice with a sham US exposure.

The treatment schematic diagram was shown in Figure 1. The treatment was performed in each group once a week for four times. After the treatment, the mice were used for the behavioral test and the histological examinations after heart perfusion. Half of the brains were fixed with $4 \%$ paraformaldehyde, and the other hemispheres were used for western blot. The diagram and time sequences of the treatment procedure were illuminated in Figure 1.

\section{Neural function evaluation with Morris Water Maze Test}

The learning and memory function were assessed in a Morris water maze (MWM, Beijing Liuyi Co. Ltd., China) task, including the place-navigation test and spatial probe test. MWM was a white-colored circular water pool in $120 \mathrm{~cm}$-diameter, $40 \mathrm{~cm}$ height and maintained at $25 \pm 2{ }^{\circ} \mathrm{C}$. The place-navigation was assessed for 5 consecutive days. A platform submerged 2 $\mathrm{cm}$ below the water surface was placed in the fourth quadrant. Mice were placed into the tank at the same point of each quadrant. If a mouse found the platform within $90 \mathrm{~s}$ and stayed on it for more than $10 \mathrm{~s}$, it was considered successful. Otherwise, the mice were manually guided to the platform and remained on it for $10 \mathrm{~s}$. The swimming paths and the time reaching the 
platform were recorded by a computerized video imaging analysis system. The interval between each trial was $60 \mathrm{~s}$.

After the final place-navigation test on the $5^{\text {th }}$ day, the spatial probe test was carried on with the platform removed. The mice were placed at any one of the four quadrants. The numbers of crossings in the area where the platform originally located and the time spending in the target quadrant were recorded for $60 \mathrm{~s}$.

\section{Identification of NSCs with immunochemistry staining}

Immunocytochemistry was performed on the neurospheres at the $3^{\text {rd }}$ passage to identify

NSCs. The neurospheres were cultured in the confocal dishes for 5 days and fixed with $4 \%$ paraformaldehyde for $5 \mathrm{~min}$ and treated with $0.1 \%$ TritonX-100 for $15 \mathrm{~min}$. After washing with PBS, the neurospheres were sequentially blocked in 10\% goat serum (Abcam, Cambridge, UK) for 10min followed by the incubation with the Anti-Nestin Antibody (Mouse monoclonal, 1:200, Millipore, MA, USA) overnight at $4{ }^{\circ} \mathrm{C}$. The next day, they were sequentially washed in PBS and incubated with Fluorescence-Conjugated affinity pure goat anti-mouse IgG (1:1000, ZSGB-Bio, Beijing, China) at $37{ }^{\circ} \mathrm{C}$ for $60 \mathrm{~min}$, followed by a washing and incubating in microscope (TCS SP5, Leica, Germany). brain-derived neurotrophic factor (BDNF) and synaptophysin (SYN) of the mice brain. After the fourth treatment, mice in each group were anesthetized and transcardially perfused with 30 $\mathrm{ml}$ NS. Then, one hemisphere of a mouse brain was extracted and fixed with $4 \%$ paraformaldehyde for $48 \mathrm{~h}$ and cut into $4 \mu \mathrm{m}$-thick paraffin slide sagittally. After dewaxing, 
hydration and antigen retrieval, the sections were sequentially treated with $0.25 \%$ TritonX-100 for $30 \mathrm{~min}$ and blocked with normal donkey serum (Abcam) for $30 \mathrm{~min}$, followed by the incubation with specific antibody of anti- $\beta$-amyloid, 1-42 (1:100, BioLegend), BDNF (rabbit polyclonal, 1:100, Proteintech) and SYN (mouse monoclonal, 1:100, Millipore). Biotinylated antibody (SP9000, ZSBG-Bio, Beijing, China) or Alexa Fluor 647-labeled Goat Anti-Rabbit $\operatorname{IgG}(1: 200$, Beyotime) was used as the secondary antibody. Five slices of each sample were observed, and the images were captured with an Olympus BX51 microscope or a Leica laser scanning confocal microscope.

\section{BDNF and SYN expression assessed with Western blotting}

Brain samples at the targeted areas were homogenized in mortar with liquid nitrogen. The proteins were extracted in the protease and phosphatase inhibitors (Beyotime) on the ice for 30 min. Bicinchoninic acid protein determination assay (BCA protein assay reagent; Beyotime) was used to analyze protein concentration. Samples $(10 \mu \mathrm{l})$ were electrophoresed in $10 \%$ sodium dodecyl sulfate (SDS)-polyacrylamide gels and transferred to nitrocellulose membranes. Then, the membranes were sequentially blocked in 5\% nonfat dry-milk solution for $1 \mathrm{~h}$, incubated with the primary antibodies of anti-BDNF (1:1000), anti-SYN (1:1000) and Tubulin (1:1000, Beyotime) overnight at $4{ }^{\circ} \mathrm{C}$. After washing the membranes with Tween-Tris-buffered saline buffer, the membranes were incubated in alkaline phosphatase-conjugated secondary antibody (1:5000, ZSGB-Bio) for $1 \mathrm{~h}$. After the incubation, the membranes were washed three times and treated with enhanced chemiluminescence method (Millipore). Images were captured with a LAS-4000 mini (GE Healthcare, Wausaukee, WI). The bands were analyzed with the signal intensity ratio between the targeted protein and Tubulin using Image $\mathrm{J}$. 
244 All the data were presented as mean values \pm standard deviation. To account for the multiple 245 mean comparisons, one-way analysis of variance (ANOVA) with least significant difference 246 (LSD) post hoc testing or nonparametric tests with Kruskal-Wallis were used. All the statistics were analyzed by SPSS 20.0 software. $P<0.05$ was considered as statistically significant.

\section{Results}

\section{Characterization of $M B_{A \beta}$}

The $\mathrm{MB}_{\mathrm{A} \beta}$ were in milky and stable at room temperature, and mainly ranging from 300 to $700 \mathrm{~nm}$ in diameter (Fig. 2D). $\mathrm{MB}_{\mathrm{A} \beta}$ can achieve the same imaging intensity as the MB with contrast-enhanced US. There were both red and green fluorescence on the membrane of MB with the fluorescence imaging, indicating FITC-labeled anti-A $\beta$-amyloid has been successfully loaded onto the DiI-labeled MB (Fig. 2A-C).

\section{Characterization of NSCs}

NSCs isolated from E12-14 C57 embryos were in free-floating neurospheres and capable of proliferation, self-renewal and differentiation. A remarkable expression of Nestin was revealed in the neurospheres with the immunofluorescence analysis (Fig. 2E, F).

\section{In vivo tracking of the survival of luciferase labeled NSCS}

In order to tracking the viability of fLuc-NSCs, in vivo imaging of mice was captured every day until no visible bioluminescence was recorded after transplantation. The results revealed none at $72 \mathrm{~h}$ (Fig. 2I). No bioluminescence was found in the other organs at any time. And the results indicated that fLuc-NSCs infusion via tail vein can go through the pulmonary circulation 
and home to the brain, and fLuc-NSCs were able to proliferate for a limited time and survive for $48 \mathrm{~h}$ in $\mathrm{AD}$ mice brain.

\section{Effect of UMMD on BBB opening}

A patchy distribution of EB extravasation was observed at the US-exposed area in the gross brain samples, while no visible EB at the unexposed area (Fig. 3A-B). Moreover, EB extravasation was visible from the activation point to the $10^{\text {th }} \mathrm{h}$ following UMMD. EB extravasation was evident in the previous $4 \mathrm{~h}$ and became weaker with time went on (Fig. 3CJ). At $10 \mathrm{~h}$, only a trace of EB extravasation could be seen in the brain parenchyma and nearly none at $12 \mathrm{~h}$. These results indicated that BBB can be opened by UMMD transiently and restorable with an opening window for no more than $12 \mathrm{~h}$. damages were detected either in the cortex or hippocampus under the light microscope, which indicated the safety of BBB opening by this method.

\section{Improved cognition performance with Morris water maze} US $+\mathrm{MB}_{\mathrm{A} \beta}+\mathrm{NSC}$ group were shorter than the other groups and had significant difference with the Control group $(P<0.05$, Fig. $4 \mathrm{~F})$. US $+\mathrm{MB}_{\mathrm{A} \beta}$ group also had significant difference when compared with the control group on day 5 ( $P<0.05$, Fig. 4F). The average time of the 5 days in the US $+\mathrm{MB}_{\mathrm{A} \beta}+\mathrm{NSCs}$ group had significant difference with Control and US+MB group but had no difference with the US+MB $\mathrm{MB}_{\mathrm{A} \beta}$ group $(P<0.05$, Fig. $4 \mathrm{G})$. In the spatial probe test, the average time had no significant difference among each groups, but the average distance in the 
target quadrant was much longer in the US $+\mathrm{MB}_{\mathrm{A} \beta}+\mathrm{NSCs}$ group than Control group (Fig. $4 \mathrm{H}$,

$P<0.01)$, and the difference between the US $+\mathrm{MB}_{\mathrm{A} \beta}$ group and Control group was less significant(Fig. $4 \mathrm{H}, P<0.05$ ). Significant difference was noted in the crosses number between the US+MB $\mathrm{MB}_{\mathrm{A}}+\mathrm{NSC}$ group and Control group (Fig. 4I, $\mathrm{P}<0.05$ ). These data indicated an improvement on the impaired learning and spatial memory of the single $A \beta$ antibody delivery and the combined implantation of $\mathrm{A} \beta$ antibody and NSCs group, especially the dual delivery group.

\section{Aß plaque reduction assessed by immunohistochemistry}

Immunohistochemistry was performed with anti- $\beta$-amyloid to investigate whether the treatments prompt the amyloid plaques clearance in the brain of the APP/PS1 (+) mice (Fig. 5A-E). A significant difference was found in the number of plaques between the $\mathrm{US}+\mathrm{MB}_{\mathrm{A} \beta}+\mathrm{NSCs}$ group and Control group both in the cortex and in the hippocampus $(P<0.05$,

Fig. 5F,G). Although no significant difference was observed among the US+MB group,

$\mathrm{US}+\mathrm{MB}_{\mathrm{A} \beta}$ group and US $+\mathrm{MB}_{\mathrm{A} \beta}+\mathrm{NSC}$ group, the number of plaques in US $+\mathrm{MB}_{\mathrm{A} \beta}+\mathrm{NSCs}$ group was fewer than the other two groups. No difference was found out between Wild group and $\mathrm{US}+\mathrm{MB}_{\mathrm{A} \beta}+\mathrm{NSC}$ group. These data showed that the NSCs transplantation combined with $\mathrm{MB}_{\mathrm{A} \beta}$ delivered by UMMD can efficiently help eliminate amyloid plaques in $\mathrm{AD}$ mice.

Elevated BDNF but not SYN expression with western blot and immunofluorescence (IF)

method

To determine whether the combined delivery approach improves the expression of BDNF and SYN, IF and western blot were performed. IF result indicating an elevated BDNF expression in the US+MB $\mathrm{MB}_{\mathrm{A}}+\mathrm{NSCs}$ group, second to that of Wild group both at the cortex and 
hippocampus (Fig. 6 A-E). In contrast, much lower level of BDNF was detected in Control group. The expression of BDNF was improved in the US+MB group and the US+MB $\mathrm{M}_{\mathrm{A}}$ group when compared to Control group, but the degree was inferior to that in the US+MB $\mathrm{A}_{\beta}+\mathrm{NSCs}$ group. Western blot analyses of BDNF were shown in Figure 6K. Much higher level of BDNF was demonstrated in the US+MB $\mathrm{MB}_{\mathrm{A}}+\mathrm{NSC}$ group than the US+MB $\mathrm{M}_{\mathrm{A} \beta}$ group and Control group $(P<0.05)$. Disappointingly, no obviously elevated expression of SYN was observed by IF (Fig. 6F-J) and western blot (Fig. 6L) in neither group. Thus, the treatment tried in the current study did not alter the level of SYN. mediated by US has recovered the expression of BDNF but not SYN in AD mice in the present study.

\section{Discussion}

$\mathrm{AD}$ affects millions of people; however, there is still no effective treatment. Therapeutic agent delivery to the brain is a potential approach for $\mathrm{AD}$ treatment. The great challenge of 
study.

BBB opening window is important on the therapeutic targets delivered into brain. BBB permeability was increased safely, transiently and restorablely in mice brain before UMMD assisted delivery treatment in the study. EB extravasation was observed to evaluate the BBB opening and lasted for no more than $12 \mathrm{~h}$ in the wild type mice following UMMD. The amount of EB extravasation reached the peak at the first time of US exposure and remained a high level in the first $4 \mathrm{~h}$, then decreased till $10 \mathrm{~h}$ and became invisible at $12 \mathrm{~h}$. In order to get the optimal delivery efficiency, MB carried $\mathrm{A} \beta$ antibody was injected during US irradiation and NSCs was infused at the first time after finishing US exposure in the study. The optimal time for the brain targeted delivery of therapeutants was no more than $4 \mathrm{~h}$ following US exposure.

The survival and the proliferation of the transplanted NSCs were vital for AD recovery. The survival of the intravenous injected NSCs in AD mice were detected with fLuc-NSCs by in vivo imaging system. Luminescence was recorded at the calvaria at $24 \mathrm{~h}, 48 \mathrm{~h}$ and $72 \mathrm{~h}$ following fLuc-NSCs injection under UMMD assisted BBB opening. The luminescence intensity was higher at $48 \mathrm{~h}$ than at $24 \mathrm{~h}$. At $72 \mathrm{~h}$, no luminescence was observed. Few studies have emphasized on the survival time of NSCs in AD with the in vivo imaging method and it is difficult to quantify the viable cell number for the methodological problems [23,24]. Our results proved that the survival time of NSCs was limited for no more than $72 \mathrm{~h}$ in AD microenvironment. The possible reason might be that the US and MB parameters tried in this study were not the best for the living and survival of NSCs in AD mice brain and should be optimized in the future study. A repeated treatment strategy of once a week for four times has been preliminarily designed and carried out. According to the limited survival time of NSCs within 
the AD brain, it is highly recommended to increase the transplantation of NSCs and therapeutic frequency to twice a week rather than once a week for 4 times.

The combined delivery approach is superior to the $A \beta$ antibody delivery assisted by UMMD on the neural functional, pathological and molecular biological improvement in $\mathrm{AD}$ mice. As demonstrated by the place-navigation test of MWM, the learning function was improved with shorter training time in both the dual delivery group and the $\mathrm{A} \beta$ antibody delivery group. In spatial probe test, the spatial memory function was greatly recovered with much longer distance in the targeted quadrant in both the dual delivery and $\mathrm{A} \beta$ antibody delivery group, and with much more crosses number in the dual delivery group compared to the control group. The neural function recover was greater in the combined delivery group than the $\mathrm{A} \beta$ antibody delivery group. All these results proved that the learning and memory function was repaired greatly both in the dual delivery group and the $\mathrm{A} \beta$ antibody delivery group, especially in the dual delivery group. Significant decrease on $A \beta$ deposition was demonstrated in the US $+\mathrm{MB}_{\mathrm{A} \beta}+\mathrm{NSCs}$ but not in $\mathrm{A} \beta$ antibody delivery group when compared to the control group at the cortex and hippocampus, and the $\mathrm{A} \beta$ plaques were fewer in the dual delivery group than $\mathrm{A} \beta$ antibody delivery group with no significant difference, which showed no superiority on $\mathrm{A} \beta$ clearance with dual delivery approach to the single delivery method. Similarly, A $\beta$ antibody delivery approach could not greatly reduce the deposition of $A \beta$ protein [24], combined with the NSCs implantation could further help the reduction of $\mathrm{A} \beta$ protein. Blurton-Jones $\mathrm{M}$ has reported that the cognitive and memory function was greatly improved without remarkable pathological changes of A $\beta$ deposition following NSCs transplantation [14]. From the similarity and difference between their study and ours results, single target of NSCs or A $\beta$ antibody could 
not greatly prompt the $\mathrm{A} \beta$ clearance, however UMMD assisted dual delivery approach played an intensifying effect on the clearance of $\mathrm{A} \beta$ plaque in $\mathrm{AD}$ mice. BDNF is a neurotrophic molecule of structural and functional integrity of the hippocampus formation, as well as the consolidation of hippocampus-dependent memory $[1,8]$. Even single target of NSCs could result in BDNF elevation and cognition function recover in $\mathrm{AD}$ mice $[13,14,21,24]$. When combined with $\mathrm{A} \beta$ antibody and assisted with UMMD, BDNF was greatly improved at the cortex and hippocampus in both the dual delivery and single delivery group with immunofluorescence (IF) and western blot analysis, and the enhancement degree in the dual targets groups was greater than the single target group. This could explain the greater efficacy on neural function recovery in the combined delivery group.

SYN is correlated with the degree of cognitive decline and the progressing of AD. Give that increasing the expression of SYN is essential. An up-regulation of BDNF was demonstrated in our result, however, there were no significant improvement in SYN with the same methods between the combined delivery group and the single $A \beta$ antibody delivery group, even though following a consecutive four times treatment. SYN was significantly reduced in relation to the deposition of $\mathrm{A} \beta$ plaques in the hippocampus and cortex in $\mathrm{AD}$, leading to the loss of synapses and dysfunction of synaptic transmission [25-27]. According to our results, $A \beta$ clearance has been improved in the dual delivery approach, while SYN remained nearly no recover. As we W. et al have reported that after 10-weeks NSCs transplantation, an increased expression of SYN and post-synaptic protein-95 were demonstrated [20]. In the present study, SYN was detected 4 weeks post-treatment, and the results might be different if a longer time about 10 
even more weeks was designed and performed. Besides, the in vivo imaging revealed that the

NSCs survived no more than $72 \mathrm{~h}$, so the effect of the implanted NSCs was weak and the effect on synaptic recover was limited. Furthermore, a non-invasive and repeatable method using intravenous infusion of NSCs was tried, and only part of NSCs was able to home to the AD brain through systemic circulation. So, the efficiency of NSCs on synaptic recover was restricted. Until now, no exact reasons are elaborated, and further study needs to be explored. While this study still has some limitations. In the future study, a much longer observation time about 10 weeks should be designed due to the restricted recovery of synaptic function and the parameters optimization should be carried out to improve NSCs survival in AD microenvironment for the limited survival time.

\section{Conclusions} could efficiently, safely increase the expression of BDNF, decrease the deposition of betaamyloid protein and restore the impaired learning and the spatial memory function when compared to single $A \beta$ antibody delivery approach, which may be a promising strategy on the treatment of AD.

\section{Abbreviations} cells; fLuc: firefly luciferase; fLuc-NSCs: Luciferase labeled NSCs; BDNF: brain-derived neurotrophic factor; SYN: synaptophysin. 


\section{Declarations}

\section{Ethics approval and consent to participate}

The research has been approved by the Institutional Animal Care and Use Committee of Army Medical University.

\section{Consent for publication}

Not applicable.

\section{Availability of data and materials}

These datasets generated and/or analyzed in the current study are available from the corresponding author on reasonable request.

\section{Competing interests}

The authors have declared that no competing interest exist.

\section{Funding}

The research was funded by grants of National Natural Science Foundation of China NSFC [81471795,81971774], the Army Cultivation Project in Medical Science and Technology [16QNP102] and the Chongqing Research Program of Basic Research and Frontier Technology [cstc2018jcyjAX0011].

\section{Authors' contributions}

Qiong Zhu was responsible for design, methodology, assemble and analysis the data, and manuscript writing. Hai Cui was responsible for design, assemble and analysis the data, and manuscript writing. Yiyi Liao, Chun Li, Xue Guan, Ying He, Yani Rong and Yi Zhang were responsible for methodology, assemble and analysis the data, and finance. Zheng Liu was responsible for supervision. Yali $\mathrm{Xu}$ was responsible for conceptualization, methodology, 
manuscript writing, review and editing, and supervision.

Acknowledgements

$443 \quad$ Not applicable.

444

445

446

447

448

449

450

451

452

453

454

455

456

457

458

459

460

461

462

463

\section{Author details}

${ }^{1}$ Department of Ultrasound, Xinqiao Hospital, Army Medical University (Third Military Medical University), Shapingba District 480037, Chongqing, China. ${ }^{2}$ Department of Ultrasound, Armed Police Force Hospital, Leshan 614300, Sichuan, China.

\section{Reference}

1. Bloom GS. Amyloid-beta and tau: the trigger and bullet in Alzheimer disease pathogenesis. Jama Neurol. 2014; 71: 505-8.

2. Blennow K, de Leon MJ, Zetterberg H. Alzheimer's disease. Lancet. 2006; 368: 387-403.

3. Burgess A, Dubey S, Yeung S, Hough O, Eterman N, Aubert I, et al. Alzheimer disease in a mouse model: MR imaging-guided focused ultrasound targeted to the hippocampus opens the blood-brain barrier and improves pathologic abnormalities and behavior. Radiology. 2014; 273: 736-45.

4. Jordao JF, Thevenot E, Markham-Coultes K, Scarcelli T, Weng YQ, Xhima K, et al. Amyloid-beta plaque reduction, endogenous antibody delivery and glial activation by brain-targeted, transcranial focused ultrasound. Exp Neurol. 2013; 248: 16-29.

5. Leinenga G, Gotz J. Scanning ultrasound removes amyloid-beta and restores memory in an Alzheimer's disease mouse model. Sci Transl Med. 2015; 7: 233r-278r.

6. Alecou T, Giannakou M, Damianou C. Amyloid beta Plaque Reduction With Antibodies Crossing the Blood-Brain Barrier, Which Was Opened in 3 Sessions of Focused Ultrasound in a Rabbit Model. J Ultrasound Med. 2017; 36: 2257-70. 
7. Jordao JF, Ayala-Grosso CA, Markham K, Huang Y, Chopra R, McLaurin J, et al. Antibodies targeted to the brain with image-guided focused ultrasound reduces amyloid-beta plaque load in the TgCRND8 mouse model of Alzheimer's disease. Plos One. 2010; 5: e10549.

8. Nisbet RM, Gotz J. Amyloid-beta and Tau in Alzheimer's Disease: Novel Pathomechanisms and Non-Pharmacological Treatment Strategies. J Alzheimers Dis. 2018; 64: S517-27.

9. Nisbet RM, Van der Jeugd A, Leinenga G, Evans HT, Janowicz PW, Gotz J. Combined effects of scanning ultrasound and a tau-specific single chain antibody in a tau transgenic mouse model. Brain. 2017; 140: 1220-30.

10. Xu M, Zhou H, Liu Y, Sun J, Xie W, Zhao P, et al. Ultrasound-Excited Protoporphyrin IX-Modified Multifunctional Nanoparticles as a Strong Inhibitor of Tau Phosphorylation and beta-Amyloid Aggregation. ACS Appl Mater Interfaces. 2018; 10: 32965-80.

11. Cui H, Zhu Q, Xie Q, Liu Z, Gao Y, He Y, et al. Low intensity ultrasound targeted microbubble destruction assists MSCs delivery and improves neural function in brain ischaemic rats. J Drug Target. 2020; 28: 320-9.

12. Xu Y, Cui H, Zhu Q, Hua X, Xia H, Tan K, et al. Unilateral Opening of Rat Blood-Brain Barrier Assisted by Diagnostic Ultrasound Targeted Microbubbles Destruction. Biomed Res Int. 2016; 2016: 4759750.

13. Blurton-Jones M, Kitazawa M, Martinez-Coria H, Castello NA, Muller FJ, Loring JF, et al. Neural stem cells improve cognition via BDNF in a transgenic model of Alzheimer disease. Proc Natl Acad Sci U S A. 2009; 106: 13594-9.

14. Zhang W, Gu GJ, Zhang Q, Liu JH, Zhang B, Guo Y, et al. NSCs promote hippocampal neurogenesis, metabolic changes and synaptogenesis in APP/PS1 transgenic mice. Hippocampus. 
15. Liu P, Wang X, Zhou S, Hua X, Liu Z, Gao Y. Effects of a novel ultrasound contrast agent with long persistence on right ventricular pressure: Comparison with SonoVue. Ultrasonics. 2011; 51: $210-4$

16. Gao S, Zhu Q, Dong X, Chen Z, Liu Z, Xie F. Guided longer pulses from a diagnostic ultrasound

21. Gu G, Zhang W, Li M, Ni J, Wang P. Transplantation of NSC-derived cholinergic neuron-like cells

20. Zhang W, Wang GM, Wang PJ, Zhang Q, Sha SH. Effects of neural stem cells on synaptic proteins and memory in a mouse model of Alzheimer's disease. J Neurosci Res. 2014; 92: 185-94. 
24. Hayashi Y, Lin HT, Lee CC, Tsai KJ. Effects of neural stem cell transplantation in Alzheimer's disease models. J Biomed Sci. 2020; $27: 29$.

25. Li X, Zhu H, Sun X, Zuo F, Lei J, Wang Z, et al. Human Neural Stem Cell Transplantation Rescues Cognitive Defects in APP/PS1 Model of Alzheimer's Disease by Enhancing Neuronal Connectivity and Metabolic Activity. Front Aging Neurosci. 2016; 8: 282.

26. Morroni F, Sita G, Tarozzi A, Rimondini R, Hrelia P. Early effects of Abeta1-42 oligomers injection in mice: Involvement of PI3K/Akt/GSK3 and MAPK/ERK1/2 pathways. Behav Brain Res. 2016; 314: 106-15.

27. Tampellini D, Capetillo-Zarate E, Dumont M, Huang Z, Yu F, Lin MT, et al. Effects of synaptic 
531 Figure 1 UMMD treatment diagram and timeframe in 5 groups of $A D$ mice

532 The US probe was kept anterior to the line of ears of the mice brain. $\mathrm{MB}_{\mathrm{AB}}, \mathrm{MB}$ and/or

533 NSCs were injected via tail vein per week for 4 times according to the groups. After the last

534 treatment, cognitive function was performed with MWM.

Figure 2 Successful MB loaded with $A \beta$ antibody, neurosphere and in vivo imaging of fLuc-

Anti- $\beta$-amyloid antibody (Green) was successfully loaded on Dil-labeled MB (Red) (A-C,

$\times 1600$ ), and the size of $M_{A_{A \beta}}$ was mainly distributed from $300 \mathrm{~nm}$ to $700 \mathrm{~nm}(\mathrm{D})$. NSCs were amassed as neurospheres at the 6th day of culture with expression of Nestin (Green, E-F, $\times 400$ ). In vivo imaging, luminescence was observed at the calvaria following $24 \mathrm{~h}$ and $48 \mathrm{~h}$ with UMMD assisted BBB opening and fLuc-NSCs implantation. The luminescence intensity was higher at $48 \mathrm{~h}(\mathrm{H})$ than at $24 \mathrm{~h}(\mathrm{G})$ and disappeared at $72 \mathrm{~h}(\mathrm{I})$.

Figure 3 EB extravasation at different time point and histological changes in wild mice

546 Strips of EB extravasation was observed in the gross views under US-exposed area (A, B).

547 After US mediated BBB opening, the degree of EB extravasation was different at different

548 time (0h, $1 \mathrm{~h}, 2 \mathrm{~h}, 4 \mathrm{~h}, 6 \mathrm{~h}, 8 \mathrm{~h}, 10 \mathrm{~h}$ and $12 \mathrm{~h}$ ), and no EB was observed after $12 \mathrm{~h}$ following

549 UMMD (C-J). No capillary injuries and erythrocyte leakages were observed in cortex (K,

$550 \times 200)$ and hippocampus $(\mathrm{L}, \times 200)$ in HE stains. 
Figure 4 The cognitive functions were analyzed with MWM after UMMD treatment hidden platform was indicated with MWM analysis $\left(F,{ }^{*}\right.$ Wild vs. Control, $\%$ US+MB $B_{A \beta}$ vs.

Control, \# US+MB ${ }_{A \beta}+N S C s$ vs. Control, $\left.P<0.05\right)$. The average time in the place-navigation test and their comparisons among 5 groups $\left(G,{ }^{*} P<0.05,{ }^{* *} P<0.01\right)$. Spatial probe test of the distance in the target quadrant was shown in the histogram $(\mathrm{H})$ : Significant difference between US $+\mathrm{MB}_{A \beta}+\mathrm{NSC}$ vs. Control ( $\left.{ }^{* *} P<0.01\right)$, less significance between US+MB $\mathrm{A}_{\mathrm{A}}$ vs. Control (* $P<0.05)$. The cross number in the target quadrant was significant different between US+MB ${ }_{A \beta}+N S C s$ vs. Control $\left(I,{ }^{*} P<0.05\right)$.

Figure 5 The histological changes in 5 groups following UMMD treatment $\times 200$ ) by immunohistochemistry. The number of amyloid plaques was counted and statistically analyzed in the hippocampus $(F)$ and cortex $(G)$, respectively. $\left({ }^{*} P<0.05\right)$.

Figure 6 The expression of BDNF and SYN with IF and western blot analysis group and US+MB $\mathrm{A}_{\beta}+\mathrm{NSC}$ group, only faintly visible expression in the other three groups.

571 Significant differences were noted between US+MB ${ }_{A \beta}+N S C s$ vs. Control, and

572 US+MB ${ }_{A \beta}+N S C s$ vs. US+MB $B_{A \beta}$ with western blot analysis $(K, P<0.05)$. No obvious difference expression of SYN (red) was demonstrated by IF (F-J, $\times 400)$ and western blot analysis (L). 
Table1: Treatment protocols in different groups

\begin{tabular}{|c|c|c|c|c|}
\hline $\operatorname{Group}(n=6)$ & US & MB $(300 \mu 1)$ & NSCs $(200 \mu 1)$ & NS $(200 \mu 1)$ \\
\hline Wild & - & - & - & + \\
\hline Control & - & - & - & + \\
\hline $\mathbf{U S}+\mathbf{M B}$ & + & MB & - & + \\
\hline $\mathbf{U S}+\mathbf{M B}_{\mathbf{A} \beta}$ & + & $\mathbf{M B}_{\mathrm{A} \beta}$ & - & + \\
\hline $\mathbf{U S}+\mathbf{M B}_{\mathrm{A} \beta}+\mathbf{N S C s}$ & + & $\mathbf{M B}_{\mathrm{A} \beta}$ & + & - \\
\hline
\end{tabular}

576 
Figures

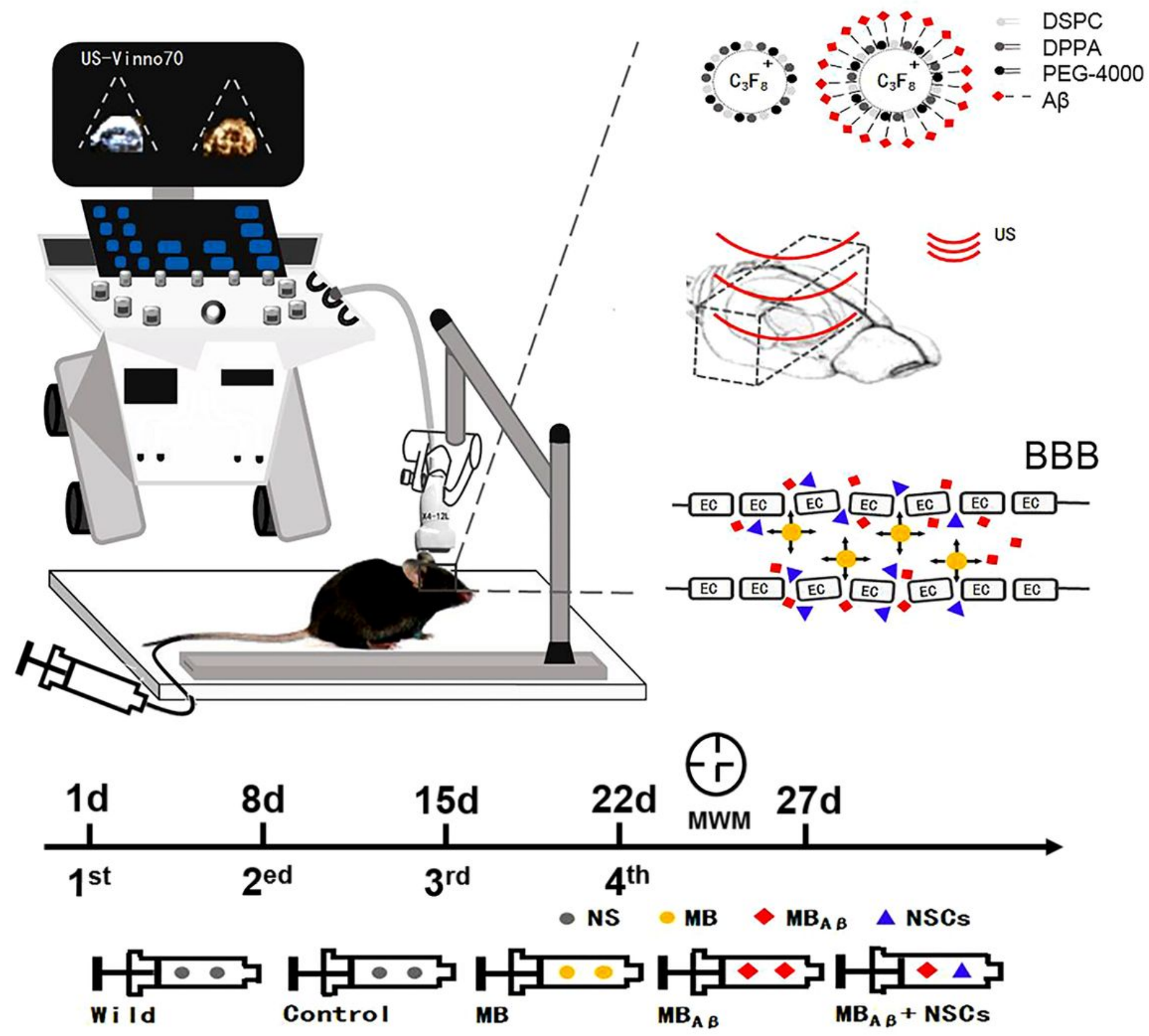

Figure 1

[See manuscript for figure legend.] 

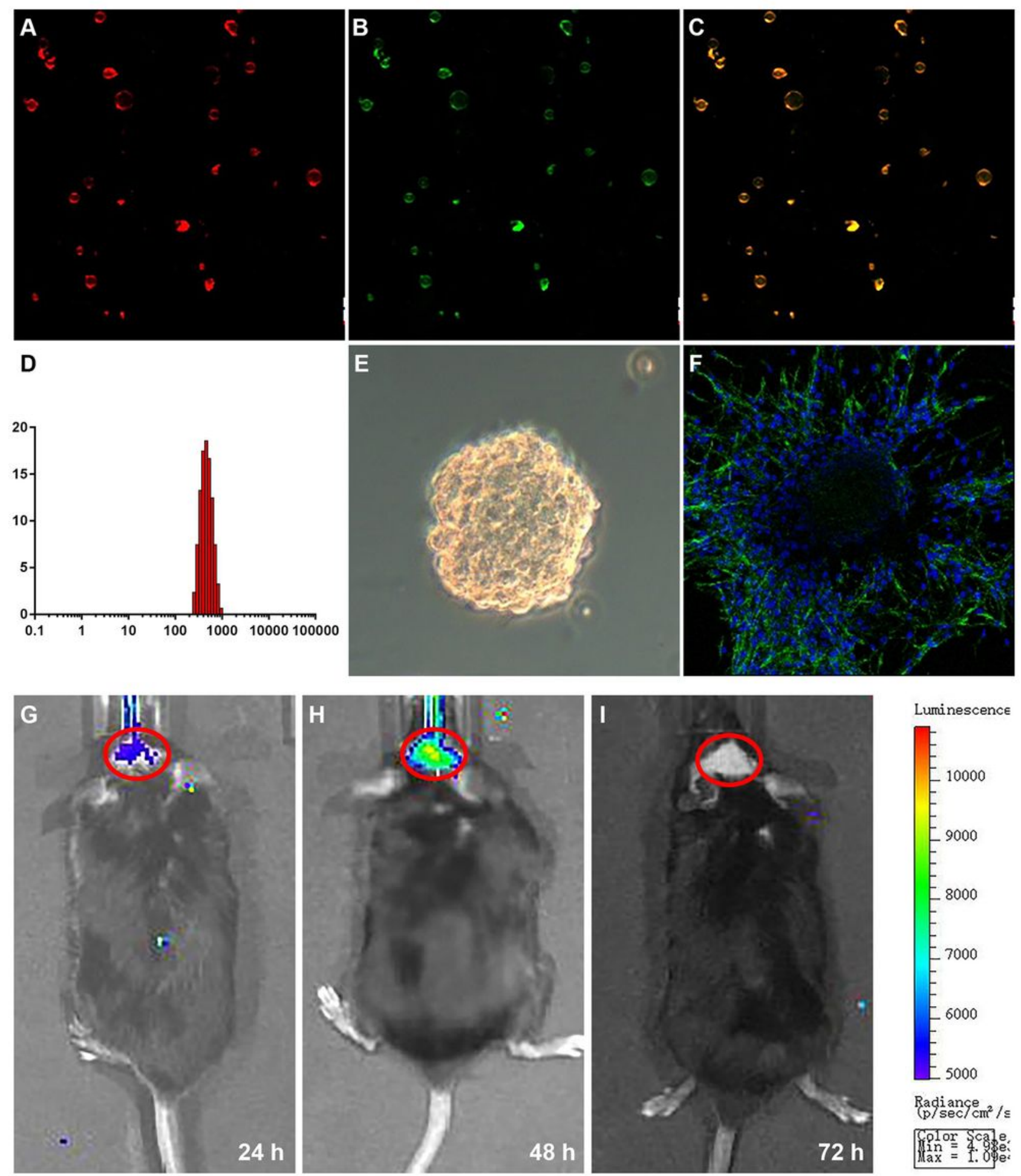

Figure 2

[See manuscript for figure legend.] 

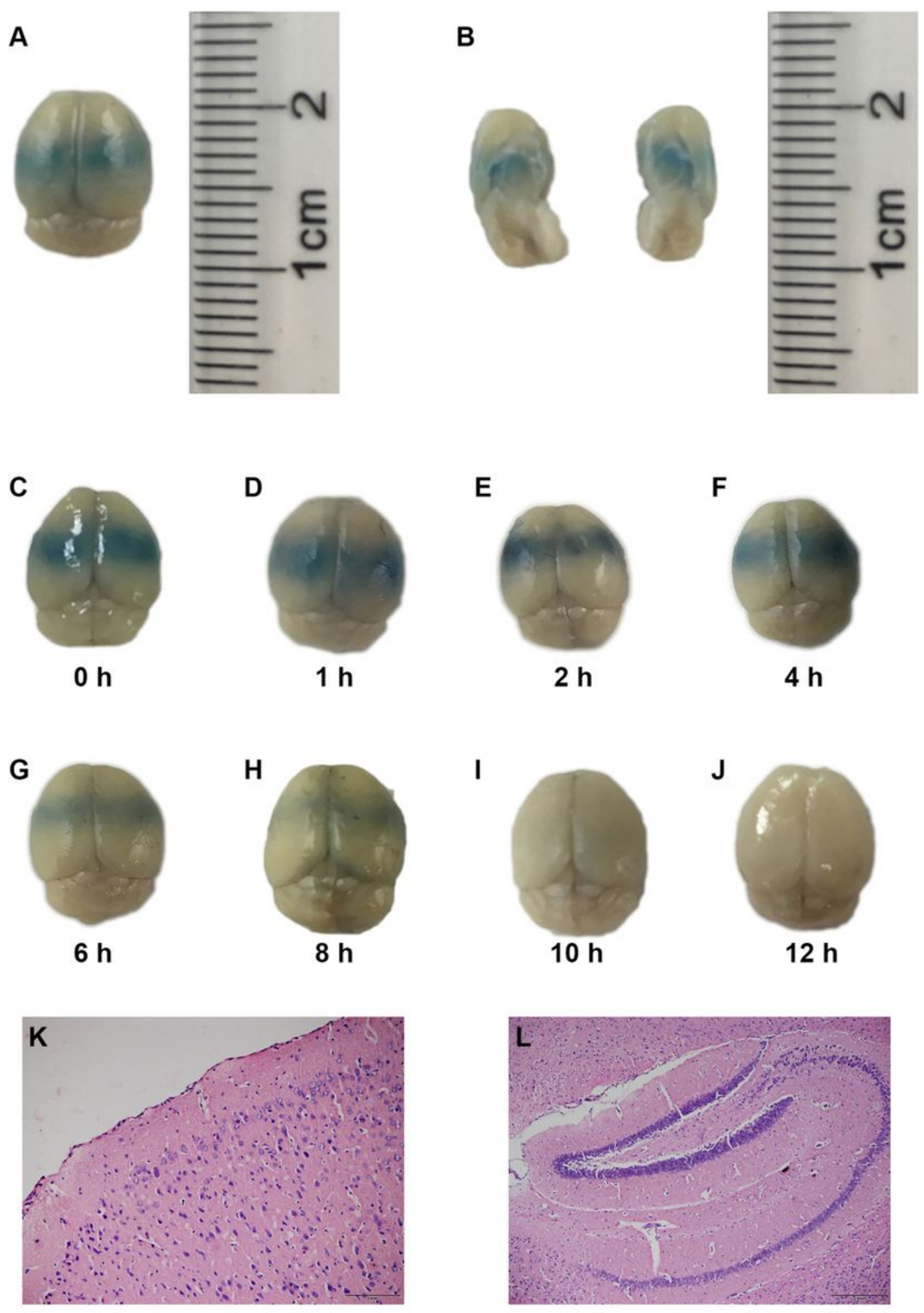

Figure 3

[See manuscript for figure legend.] 

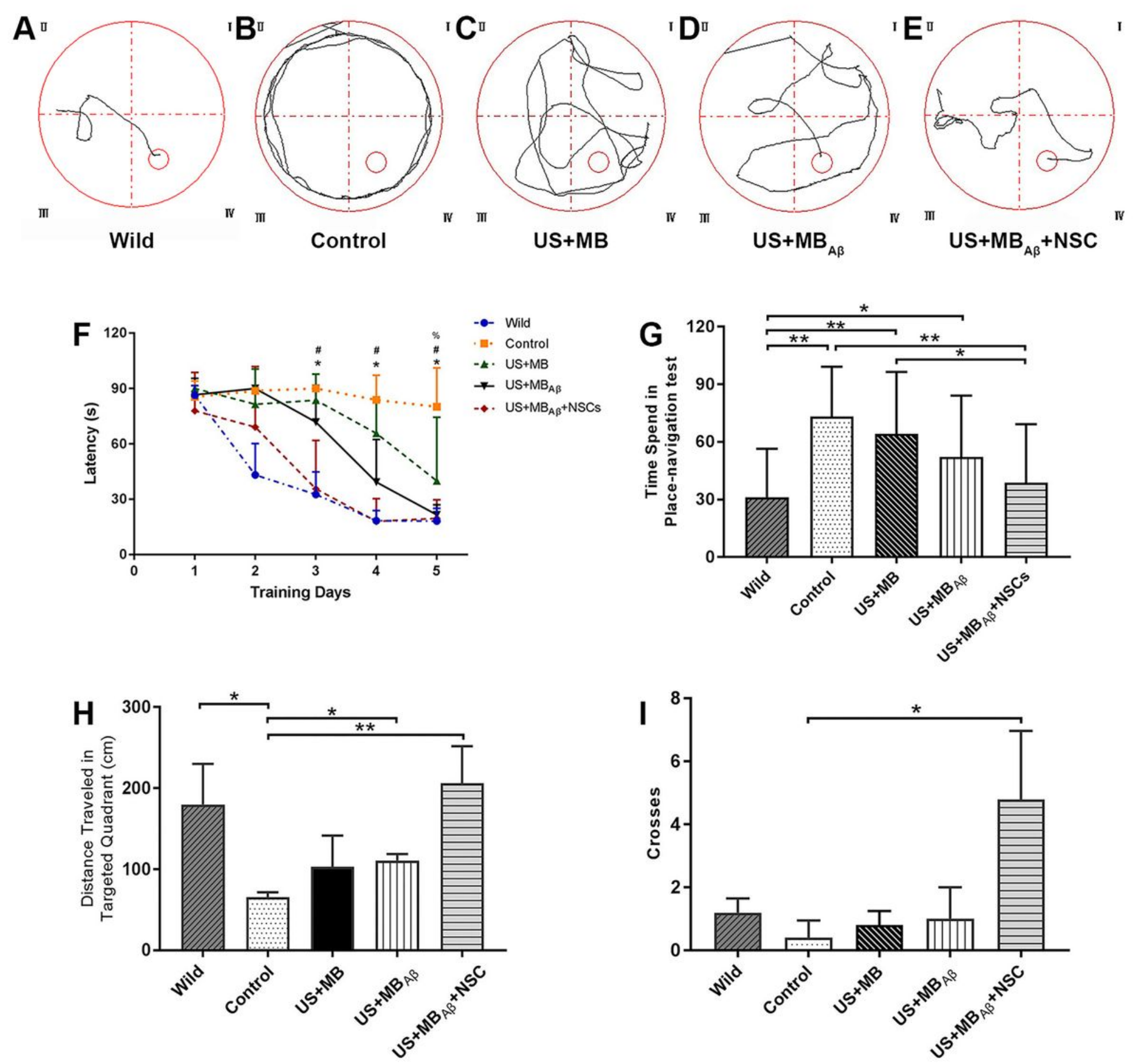

Figure 4

[See manuscript for figure legend.] 

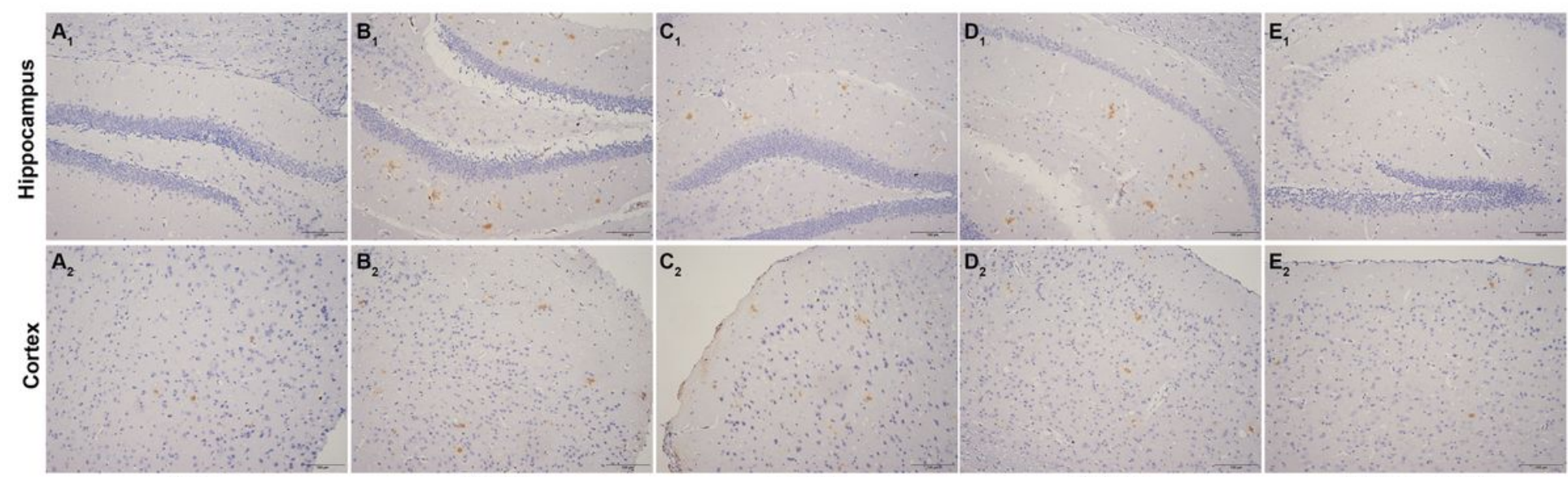

Wild

Control

Hippocampus

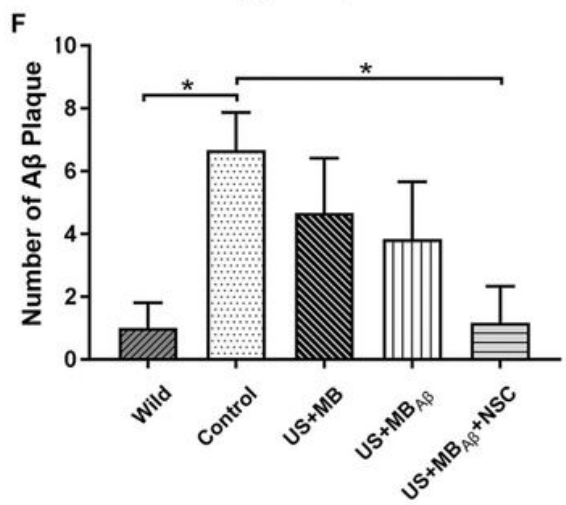

US+MB

$\mathrm{US}+\mathrm{MB}_{\mathrm{AB}}$

$\mathrm{US}+\mathrm{MB}_{\mathrm{A \beta}}+\mathrm{NSC}$

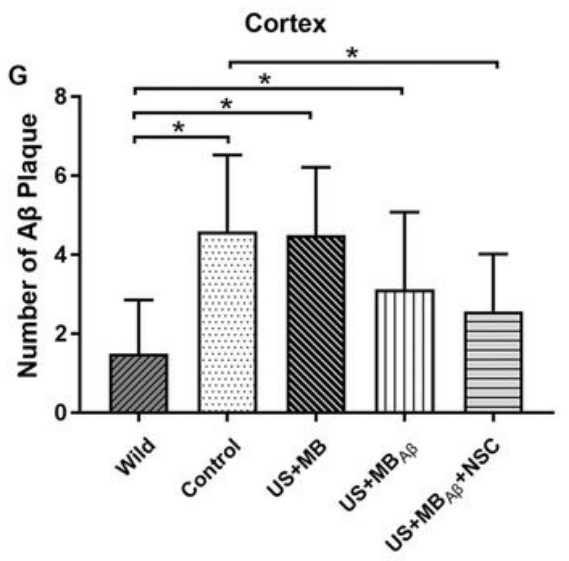

Figure 5

[See manuscript for figure legend.] 

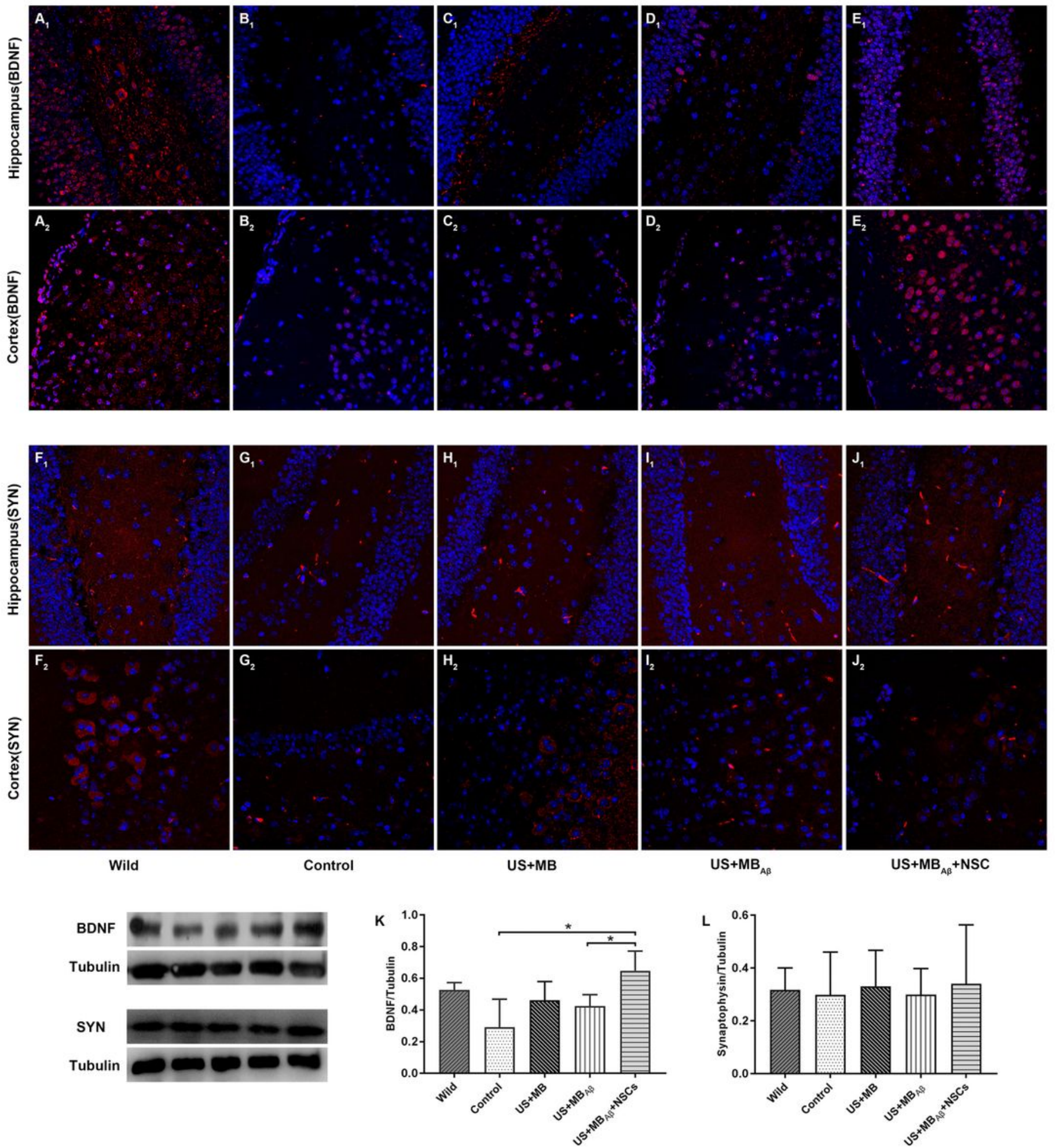

Figure 6

[See manuscript for figure legend.]

\section{Supplementary Files}

This is a list of supplementary files associated with this preprint. Click to download. 
- Table.pdf 